\title{
Characterization of inbred corn lineages for resistance to the fall armyworm based on non-feeding preference and antibiosis
}

B.E.A. Silva, W.S.C. Candido, M.L Costa, A.O. Smaniotto, J.P. Maia F.S. Gielfi and EF Reis

Universidade Federal de Goiás, Unidade Jatobá, Jataí, Goiás, Brasil

Corresponding author: B.E.A. Silva

E-mail: brunaelainea@gmail.com

Genet. Mol. Res. 19 (4): gmr18446

Received July 24, 2019

Accepted October 23, 2020

Published November 30, 2020

DOI http://dx.doi.org/10.4238/gmr18446

\begin{abstract}
One of the pests that infests the corn crop (Zea mays) in Brazil is the fall armyworm (Spodoptera frugiperda, Lepdoptera: Noctuidae), considered its most important pest because of the significant damage produced. The use of resistant cultivars is an efficient control method. The objective of this work was to evaluate the mechanisms of resistance of non-feeding preference and antibiosis of newly hatched caterpillars on leaves of inbred corn lineages, derived from populations of commercial hybrid origins, Creole populations and semi-exotic populations. The no-choice non-feeding preference test was conducted in the laboratory, with three replications per 24 hours, using leaves of 169 corn lineages. For the antibiosis test, lineages that had a smaller leaf area consumed were selected and also lineages that had a larger leaf area consumed, which were sown in a greenhouse to proceed with leaf harvesting and the tests. Newly hatched larvae of $S$, frugiperda were fed daily, through the provision of leaves of the lineages evaluated with regard to biomass and length after 5,10 and 15 days feeding. Besides presenting a non-feeding preference mechanism of resistance, lineages 94 and 137 also led to a change in the development of caterpillars, being promising to increase the efficiency of resistant cultivars in breeding programs.
\end{abstract}

Key words: Spodoptera frugiperda; Mechanisms of resistance; Zea mays; Non-feeding preference; Antibiosis 


\section{INTRODUCTION}

The corn crop (Zea mays) is one of the most consumed and cultivated cereals worldwide, and this demand is justified due to several applications in the agribusiness sector (Cardoso et al., 2011; Shiferaw et al., 2011). Regarded as one of the most produced grains globally, the estimated production for the 2018/19 harvest is 1,098.95 million tons, according to the United States Department of Agriculture (USDA, 2018).

Along with production growth, the incidence of pests in the corn crop is one of the factors that determines the profitability of the producer, especially the key pest for the crop, the fall armyworm (Spodoptera frugiperda, Lepidoptera: Noctuidae) (Barros et al., 2010). Severe damage to the cob and leaves resulting from their feeding can compromise crop yield by over $50 \%$, thus requiring integrated management strategies to reduce the population level of the pest below damage levels (Cruz, 2008).

The use of genetically modified plants expressing the insecticide gene Bacillus thuringiensis (Bt), allows a reduction in the number of applications; however, the evolution of resistance of the armyworm has compromised the success of these bioinsecticides in the field (Waquil et al., 2016). Given this need, genetic improvement programs exploring the selection of populations with a certain level of resistance are alternatives for the development of resistant pest cultivars (Reis and Miranda Filho, 2003), using the measurement of resistance mechanisms classified as non-feeding preference and antibiosis, which occur when the plant is less used by the insect for feeding, oviposition or shelter, and when the plant has the capacity to cause harmful effects on the biology of the pest, respectively (War et al., 2012; Seifi et al., 2013).

The use of resistant plants is a desirable strategy because in addition to allowing the reduction of considerable losses in production, they are compatible with other control strategies, do not increase production costs, do not pose risk to human or animal health, and do not pollute the environment (Lara, 1991; Janini et al., 2011; Jesus et al., 2014). Thus, sustainable plant protection practices contribute to the equilibrium of integrated pest management primarily for family farmers, in search of a sustainable and organic agriculture.

The success in the development of resistant cultivars depends on the efficiency of the selection of parents to be used in crosses which will originate them (Rodrigues et al., 2009). Selection performed in inbred lines, in which the level of homozygosity is high, indicating gene fixation, allows the generation of plants with identical genotypes (Falconer, 1982). For this reason, these lineages are carried to homozygosity through successive selffertilizations in order to be used in crosses, aiming at obtaining hybrids with the phenotype of interest (Silveira and Moro, 2009).

According to Alvarez and Miranda Filho (2002), despite the high variability of corn germplasm in Brazil, the identification of new sources of resistance is extremely important due to the increase of insect attacks, which would allow the introduction of new genes in commercial cultivars or even the identification of previously unknown resistance mechanisms or genes, through the use of new genotypes. Thus, the objective of this work was to evaluate the mechanisms of resistance of non-feeding preference and antibiosis of newly hatched larvae of $S$. frugiperda for leaves of corn lineages originated from populations from commercial hybrids, creole populations and semi-exotic populations. 


\section{MATERIAL AND METHODS}

\section{Feeding non-preference test by $S$. frugiperda}

The no-choice feeding non-preference test was conducted in the laboratory, in a completely randomized design with three replications. A total of 169 inbred maize lineages were planted in the field, obtained by self-fertilization of populations from commercial hybrids, creole populations and semi-exotic populations (Table 1). From these, leaves were collected at 40 days after sowing, which were sent to the laboratory and previously washed in distilled water, with excess water being removed with paper towels. From each lineage, two leaf discs were obtained, with the aid of a drill with a $3.5 \mathrm{~cm}^{2}$ area. Both discs were identified and weighed to obtain fresh weight.

Table 1. Origin, codes, generation and description of the inbred corn lines tested.

\begin{tabular}{|c|c|c|c|}
\hline Origin & Codes & Generation & Description \\
\hline MPA & 1 to 8 and 23 to 25 & S5 & $\begin{array}{l}\text { Population of creole origin, originated from } \\
\text { movement of small farmer - MPA }\end{array}$ \\
\hline SIMPA & 26 to 48 & S4 & Synthetic origin of the population MPA \\
\hline HG49 & 16 to 22 & S4 & $\begin{array}{l}\text { Population originated from hybrid already } \\
\text { withdrawn from the market in Goiás State, Brazil } \\
\text { Interpopulation hybrid population of the project }\end{array}$ \\
\hline NAPPM x HG49 & 9 to 15 & S4 & $\begin{array}{l}\text { NAP with resistance to Phaeosphaeria } \\
\text { maydis and hybrid HG49 }\end{array}$ \\
\hline RV CERC & $\begin{array}{l}49 \text { to } 51 ; 62 \text { to } 65 ; 71 \text { to } 86 \text {; } \\
93 ; 135 \text { to } 143\end{array}$ & S4 & $\begin{array}{l}\text { Hybrid selected for resistance to } \\
\text { cercospora originated from hybrids } \\
\text { withdrawn from the market in Goiás State, Brazil }\end{array}$ \\
\hline SUPERPRECOCE & $60 ; 118$ to 124 & S5 & Very early population \\
\hline 2B587 & 52 to $59 ; 87$ to $92 ; 94$ to 117 & S5 & Commercial hybrid planted in Goiás State, Brazil \\
\hline AG7088 & 66 to $70 ; 155$ to 169 & S5 & Commercial hybrid planted in Goiás State, Brazil \\
\hline 2B433 & $61 ; 125$ to 134 & S5 & Commercial hybrid planted in Goiás State, Brazil \\
\hline AG6040 & 144 to 154 & S5 & Commercial hybrid planted in Goiás State, Brazil \\
\hline
\end{tabular}

Subsequently, one of the discs was placed in a Petri dish for consumption by the newly hatched $S$. frugiperda caterpillars from laboratory breeding, with one disc from each lineage individualized in one plate, with three caterpillars in the center of each plate, feeding for 24 hours. Petri dishes were kept in an incubator under controlled conditions of temperature $\left(25 \pm 1^{\circ} \mathrm{C}\right)$, relative humidity $(70 \pm 15 \%)$ and photophase (14 hours). After this period, the leftover discs were removed and placed in a constant weight oven to determine the final dry mass.

The other leaf disc was directly placed to dry in an oven at $60^{\circ} \mathrm{C}$ until reaching constant mass, allowing the calculation of the dry mass of the original disc, without consumption. The dry mass consumed was calculated using the following equation:

$$
D M C=\left\{\left[1-\left(\frac{M F O-D M O}{M F O}\right)\right] \times M F C\right\}-M S C
$$

Where:

DMC: dry matter consumed

MFO: fresh mass of the original disc that was not delivered to the caterpillars

MFC: fresh mass of the disk delivered to the caterpillars in the Petri plate 
DMO: dry mass of the original disk

MSC: dry mass of disks leftovers after 24 hours consumption

And the leaf area consumed was given by:

$$
L A C=3.5 \times\left(\frac{D M C}{D M D}\right)
$$

Where:

AREA OF THE DISK $-3.5 \mathrm{~cm}^{2}$

DMC: dry matter consumed

DMD: dry matter of the disk supplied to the caterpillars, given by:

$$
D M D=\left\{\left[1-\left(\frac{M F O-D M O}{M F O}\right)\right] \times M F C\right\}
$$

\section{Antibiosis Test}

The lineages submitted to the antibiosis test were selected a priori on the basis of the results obtained by no-choice feeding non-preference, and according to the availability of seeds in the germplasm bank. Thus, 24 lineages least preferred for feeding by newly hatched $S$. frugiperda larvae, and 5 most preferred lineages were selected (Table 2).

Table 2. Identification of selected corn lineages in the no-choice non-feeding preference test, intended for

\begin{tabular}{|c|c|c|}
\hline Codes of selected strains & Origin & Classification \\
\hline 4 & MPA & Less preferred \\
\hline 6 & MPA & Less preferred \\
\hline 21 & HG49 & Less preferred \\
\hline 23 & MPA & Less preferred \\
\hline 27 & SIMPA & Less preferred \\
\hline 28 & SIMPA & Less preferred \\
\hline 30 & SIMPA & Less preferred \\
\hline 62 & RV CERC. & Less preferred \\
\hline 65 & RV CERC. & Less preferred \\
\hline 70 & AG 7088 & Less preferred \\
\hline 77 & RV CERC. & Less preferred \\
\hline 78 & RV CERC. & Less preferred \\
\hline 86 & RV CERC. & Less preferred \\
\hline 87 & 2B587 & Less preferred \\
\hline 88 & 2B587 & Less preferred \\
\hline 91 & 2B587 & Less preferred \\
\hline 94 & 2B587 & Less preferred \\
\hline 96 & 2B587 & Less preferred \\
\hline 97 & 2B587 & Less preferred \\
\hline 98 & 2B587 & Less preferred \\
\hline 99 & 2B587 & Less preferred \\
\hline 100 & 2B587 & Less preferred \\
\hline 137 & RV CERC. & Less preferred \\
\hline 161 & AG 7088 & More preferred \\
\hline 123 & SUPERPRECOCE & More preferred \\
\hline 110 & 2B587 & More preferred \\
\hline 134 & 2B433 & More preferred \\
\hline 74 & RV CERC. & More preferred \\
\hline
\end{tabular}
the realization of the antibiosis test. 
In order to maintain the feeding of newly hatched caterpillars used for the antibiosis test, the selected corn lineages were planted in 14-liter plastic pots filled with soil, fertilized and the $\mathrm{pH}$ was corrected, according to the recommendations for the corn crop. Three pots were used for each treatment, with three plants per pot arranged in a greenhouse.

The experiment was carried out in a completely randomized design, in a split plot scheme, in the laboratory, where the newly-hatched caterpillars were individualized in Petri plates coated with moistened filter paper, in four replicates per lineage, with each caterpillar corresponding to a replicate. They were fed daily with $14 \mathrm{~cm}^{2}$ of leaves of selected corn inbred lineages, which were properly washed and excess water removed before being supplied to the caterpillars. Regardless of the consumption of leaves by caterpillars, feed with the leaves were changed daily, after washing of the plates.

Evaluations were conducted 5,10 and 15 days after the outbreak of caterpillars and posterior feeding, where the following parameters were assessed:

Biomass of caterpillars: obtained through the individual weighing of each caterpillar of each treatment using a precision scale and expressed in grams. millimeters.

Length of the caterpillars: performed with the aid of a pachymeter and expressed in

The data were subjected to analysis of variance ( $F$ test) with 5\% significance and the means were grouped by Scott-Knott test using the R software (R core team, 2018).

\section{RESULTS}

The strains submitted to the no-choice feeding non-preference test showed significant differences among themselves in dry matter consumed (Table 3) and leaf area consumed (Table 4), according to the grouping of the means by the Scott-Knott procedure.

The less consumed lineages were 4, 6, 21, 23, 27, 28, 30, 42, 62, 65, 70, 77, 78, 82, $84,86,87,88,91,93,94,95,96,97,98,99,100$ and 137 , which primarily belonged to the original groups MPA, SIMPA, RVCERC and 2B587. This is due to the characteristics present in these genotypes, which may behave like stimulants or repellents in the insect feeding preference, defining the insect-plant interaction (Boiça Junior et al., 2011). This repellent effect can be explained by the volatilization of secondary compounds from leaves (Vendramim and Guzzo, 2009).

The cause of this resistance is possibly a result of the production of chemical compounds by plants, in order to protect themselves against herbivores (Pinto-Zevallos et al., 2013). Another possibility is the formation of physical barriers, such as the smooth surface of the cuticle and epidermis, hardness of the leaf, the presence of crystals and trichomes that may have irritant properties to phytophagous insect, or may cause repellence, immobility or death (Levin, 1973; Lara, 1991).

According to Ota et al. (2011), the use of cultivars with these characteristics may assist in the maintenance of the pest population at moderate levels, in combination with chemical control, because according to Wiseman and Davis (1979) the variety that is not preferred when grown alone is the most valuable source of resistance, providing inputs on the identification and quantification of resistance mechanisms.

Table 5 indicates that in the antibiosis test there was an interaction between the variables lineages and time for larvae weight. After five days of feeding, the caterpillars fed with strains 98, 74, 27, 65, 30, 6, 91, 110, 86, 21, 100, 96, 87, 161, 99, 62, 28, 78, 88, 94 
and 137 presented lower mean weight compared to those fed with other materials. However, after ten days feeding the mean values observed were similar in all strains. After 15 days feeding, only the lineage 94 originating from cultivar 2B587 and 137 from RVCERC led to lower weights of caterpillars.

Table 3. Means of 169 corn lineages subjected to the no-choice feeding non-preference test by Spodoptera frugiperda, evaluated by the dry mass consumed, in grams, in 24 hours.

\begin{tabular}{|c|c|c|c|c|c|c|c|}
\hline LIN & DMC & LIN & DMC & LIN & DMC & LIN & DMC \\
\hline 161 & $0.007467 \mathrm{a}$ & 135 & $0.003100 \mathrm{~b}$ & 139 & $0.001100 \mathrm{c}$ & 43 & $0.000266 \mathrm{c}$ \\
\hline 151 & $0.006800 \mathrm{a}$ & 41 & $0.003100 \mathrm{~b}$ & 160 & $0.001066 \mathrm{c}$ & 166 & $0.000266 \mathrm{c}$ \\
\hline 110 & $0.006600 \mathrm{a}$ & 159 & $0.002833 \mathrm{~b}$ & 22 & $0.001066 \mathrm{c}$ & 29 & $0.000233 \mathrm{c}$ \\
\hline 134 & $0.006466 \mathrm{a}$ & 149 & $0.002500 \mathrm{c}$ & 60 & $0.001033 \mathrm{c}$ & 81 & $0.000233 \mathrm{c}$ \\
\hline 63 & $0.006366 \mathrm{a}$ & 58 & $0.002433 \mathrm{c}$ & 48 & $0.001000 \mathrm{c}$ & 33 & $0.000166 \mathrm{c}$ \\
\hline 118 & $0.006366 \mathrm{a}$ & 112 & $0.002366 \mathrm{c}$ & 18 & $0.000966 \mathrm{c}$ & 5 & $0.000133 \mathrm{c}$ \\
\hline 123 & $0.006366 \mathrm{a}$ & 75 & $0.002300 \mathrm{c}$ & 67 & $0.000966 \mathrm{c}$ & 9 & $0.000133 \mathrm{c}$ \\
\hline 125 & $0.005700 \mathrm{a}$ & 156 & $0.002166 \mathrm{c}$ & 122 & $0.000966 \mathrm{c}$ & 64 & $0.000133 \mathrm{c}$ \\
\hline 155 & $0.005700 \mathrm{a}$ & 15 & $0.002133 \mathrm{c}$ & 71 & $0.000933 \mathrm{c}$ & 50 & $0.000100 \mathrm{c}$ \\
\hline 115 & $0.005633 \mathrm{a}$ & 106 & $0.002133 \mathrm{c}$ & 13 & $0.000900 \mathrm{c}$ & 85 & $0.000100 \mathrm{c}$ \\
\hline 74 & $0.005100 \mathrm{a}$ & 111 & $0.002100 \mathrm{c}$ & 152 & $0.000900 \mathrm{c}$ & 52 & $0.000066 \mathrm{c}$ \\
\hline 114 & $0.004933 \mathrm{~b}$ & 55 & $0.002066 \mathrm{c}$ & 59 & $0.000866 \mathrm{c}$ & 61 & $0.000033 \mathrm{c}$ \\
\hline 133 & $0.004766 \mathrm{~b}$ & 80 & $0.002066 \mathrm{c}$ & 37 & $0.000800 \mathrm{c}$ & 4 & $0.000000 \mathrm{c}$ \\
\hline 113 & $0.004666 \mathrm{~b}$ & 7 & $0.002033 \mathrm{c}$ & 158 & $0.000800 \mathrm{c}$ & 6 & $0.000000 \mathrm{c}$ \\
\hline 124 & $0.004666 \mathrm{~b}$ & 105 & $0.001900 \mathrm{c}$ & 45 & $0.000766 \mathrm{c}$ & 21 & $0.000000 \mathrm{c}$ \\
\hline 57 & $0.004600 \mathrm{~b}$ & 90 & $0.001866 \mathrm{c}$ & 83 & $0.000766 \mathrm{c}$ & 23 & $0.000000 \mathrm{c}$ \\
\hline 69 & $0.004166 \mathrm{~b}$ & 26 & $0.001833 \mathrm{c}$ & 35 & $0.000666 \mathrm{c}$ & 27 & $0.000000 \mathrm{c}$ \\
\hline 117 & $0.004000 \mathrm{~b}$ & 108 & $0.001833 \mathrm{c}$ & 49 & $0.000633 \mathrm{c}$ & 28 & $0.000000 \mathrm{c}$ \\
\hline 121 & $0.004000 \mathrm{~b}$ & 107 & $0.001800 \mathrm{c}$ & 104 & $0.000633 \mathrm{c}$ & 30 & $0.000000 \mathrm{c}$ \\
\hline 147 & $0.003933 \mathrm{~b}$ & 163 & $0.001766 \mathrm{c}$ & 39 & $0.000600 \mathrm{c}$ & 42 & $0.000000 \mathrm{c}$ \\
\hline 167 & $0.003933 \mathrm{~b}$ & 1 & $0.001733 \mathrm{c}$ & 89 & $0.000600 \mathrm{c}$ & 62 & $0.000000 \mathrm{c}$ \\
\hline 127 & $0.003833 \mathrm{~b}$ & 17 & $0.001700 \mathrm{c}$ & 144 & $0.000600 \mathrm{c}$ & 65 & $0.000000 \mathrm{c}$ \\
\hline 116 & $0.003800 \mathrm{~b}$ & 24 & $0.001666 \mathrm{c}$ & 102 & $0.000566 \mathrm{c}$ & 70 & $0.000000 \mathrm{c}$ \\
\hline 162 & $0.003800 \mathrm{~b}$ & 73 & $0.001600 \mathrm{c}$ & 2 & $0.000566 \mathrm{c}$ & 77 & $0.000000 \mathrm{c}$ \\
\hline 10 & $0.003766 \mathrm{~b}$ & 31 & $0.001566 \mathrm{c}$ & 38 & $0.000533 \mathrm{c}$ & 78 & $0.000000 \mathrm{c}$ \\
\hline 126 & $0.003733 \mathrm{~b}$ & 19 & $0.001566 \mathrm{c}$ & 145 & $0.000533 \mathrm{c}$ & 82 & $0.000000 \mathrm{c}$ \\
\hline 12 & $0.003666 \mathrm{~b}$ & 66 & $0.001566 \mathrm{c}$ & 32 & $0.000500 \mathrm{c}$ & 84 & $0.000000 \mathrm{c}$ \\
\hline 164 & $0.003633 \mathrm{~b}$ & 14 & $0.001533 \mathrm{c}$ & 47 & $0.000500 \mathrm{c}$ & 86 & $0.000000 \mathrm{c}$ \\
\hline 76 & $0.003600 \mathrm{~b}$ & 109 & $0.001533 \mathrm{c}$ & 51 & $0.000500 \mathrm{c}$ & 87 & $0.000000 \mathrm{c}$ \\
\hline 148 & $0.003566 \mathrm{~b}$ & 157 & $0.001533 \mathrm{c}$ & 138 & $0.000500 \mathrm{c}$ & 88 & $0.000000 \mathrm{c}$ \\
\hline 150 & $0.003566 \mathrm{~b}$ & 101 & $0.001500 \mathrm{c}$ & 143 & $0.000500 \mathrm{c}$ & 91 & $0.000000 \mathrm{c}$ \\
\hline 129 & $0.003533 \mathrm{~b}$ & 119 & $0.001500 \mathrm{c}$ & 56 & $0.000466 \mathrm{c}$ & 93 & $0.000000 \mathrm{c}$ \\
\hline 169 & $0.003533 \mathrm{~b}$ & 3 & $0.001466 \mathrm{c}$ & 72 & $0.000466 \mathrm{c}$ & 94 & $0.000000 \mathrm{c}$ \\
\hline 132 & $0.003466 \mathrm{~b}$ & 44 & $0.001400 \mathrm{c}$ & 165 & $0.000466 \mathrm{c}$ & 95 & $0.000000 \mathrm{c}$ \\
\hline 120 & $0.003433 \mathrm{~b}$ & 20 & $0.001266 \mathrm{c}$ & 36 & $0.000400 \mathrm{c}$ & 96 & $0.000000 \mathrm{c}$ \\
\hline 146 & $0.003433 \mathrm{~b}$ & 53 & $0.001233 \mathrm{c}$ & 46 & $0.000400 \mathrm{c}$ & 97 & $0.000000 \mathrm{c}$ \\
\hline 25 & $0.003333 \mathrm{~b}$ & 8 & $0.001200 \mathrm{c}$ & 154 & $0.000400 \mathrm{c}$ & 98 & $0.000000 \mathrm{c}$ \\
\hline 128 & $0.003300 \mathrm{~b}$ & 54 & $0.001200 \mathrm{c}$ & 16 & $0.000366 \mathrm{c}$ & 99 & $0.000000 \mathrm{c}$ \\
\hline 130 & $0.003266 \mathrm{~b}$ & 79 & $0.001166 \mathrm{c}$ & 103 & $0.000333 \mathrm{c}$ & 100 & $0.000000 \mathrm{c}$ \\
\hline 68 & $0.003233 \mathrm{~b}$ & 153 & $0.001166 \mathrm{c}$ & 168 & $0.000333 \mathrm{c}$ & 137 & $0.000000 \mathrm{c}$ \\
\hline 136 & $0.003233 \mathrm{~b}$ & 140 & $0.001133 \mathrm{c}$ & 34 & $0.000300 \mathrm{c}$ & & \\
\hline 142 & $0.003200 \mathrm{~b}$ & 141 & $0.001133 \mathrm{c}$ & 40 & $0.000300 \mathrm{c}$ & & \\
\hline 131 & $0.003166 \mathrm{~b}$ & 11 & $0.001100 \mathrm{c}$ & 92 & $0.000300 \mathrm{c}$ & & \\
\hline
\end{tabular}

Means followed by the same letter in column do not differ at $5 \%$ significance by the Scott-Knott test. LIN: lineages; DMC: dry mass consumed $(\mathrm{g})$, ordered by consumption 
Table 4. Means of 169 corn lineages subjected to the no-choice feeding non-preference test by Spodoptera frugiperda, evaluated by leaf area consumed, in $\mathrm{cm}^{2}$ in 24 hours.

\begin{tabular}{|c|c|c|c|c|c|c|c|}
\hline LIN & AFC & LIN & AFC & LIN & AFC & LIN & AFC \\
\hline 161 & $2.0491 \mathrm{a}$ & 131 & $0.8445 \mathrm{~b}$ & 54 & $0.3286 \mathrm{c}$ & 34 & $0.0836 \mathrm{c}$ \\
\hline 110 & $1.9892 \mathrm{a}$ & 132 & $0.7918 \mathrm{~b}$ & 141 & $0.3222 \mathrm{c}$ & 43 & $0.0752 \mathrm{c}$ \\
\hline 151 & $1.9381 \mathrm{a}$ & 58 & $0.7853 \mathrm{~b}$ & 13 & $0.3117 \mathrm{c}$ & 29 & $0.0723 \mathrm{c}$ \\
\hline 123 & $1.9018 \mathrm{a}$ & 80 & $0.7530 \mathrm{~b}$ & 153 & $0.3089 \mathrm{c}$ & 81 & $0.0614 \mathrm{c}$ \\
\hline 63 & $1.8268 \mathrm{a}$ & 75 & $0.7466 \mathrm{~b}$ & 48 & $0.2950 \mathrm{c}$ & 33 & $0.0526 \mathrm{c}$ \\
\hline 134 & $1.7743 \mathrm{a}$ & 112 & $0.7068 \mathrm{c}$ & 18 & $0.2942 \mathrm{c}$ & 64 & $0.0386 \mathrm{c}$ \\
\hline 155 & $1.7126 \mathrm{a}$ & 149 & $0.6657 \mathrm{c}$ & 60 & $0.2826 \mathrm{c}$ & 5 & $0.0376 \mathrm{c}$ \\
\hline 118 & $1.6567 \mathrm{a}$ & 7 & $0.6572 \mathrm{c}$ & 139 & $0.2814 \mathrm{c}$ & 9 & $0.0338 \mathrm{c}$ \\
\hline 115 & $1.6084 \mathrm{a}$ & 41 & $0.6551 \mathrm{c}$ & 67 & $0.2486 \mathrm{c}$ & 85 & $0.0328 \mathrm{c}$ \\
\hline 114 & $1.5727 \mathrm{a}$ & 106 & $0.6348 \mathrm{c}$ & 122 & $0.2392 \mathrm{c}$ & 50 & $0.0253 \mathrm{c}$ \\
\hline 133 & $1.5508 \mathrm{a}$ & 156 & $0.6001 \mathrm{c}$ & 37 & $0.2368 \mathrm{c}$ & 52 & $0.0179 \mathrm{c}$ \\
\hline 74 & $1.5116 \mathrm{a}$ & 111 & $0.5802 \mathrm{c}$ & 152 & $0.2349 \mathrm{c}$ & 61 & $0.0057 \mathrm{c}$ \\
\hline 125 & $1.3751 \mathrm{a}$ & 55 & $0.5621 \mathrm{c}$ & 59 & $0.2332 \mathrm{c}$ & 4 & $0.0000 \mathrm{c}$ \\
\hline 69 & $1.3717 \mathrm{a}$ & 90 & $0.5530 \mathrm{c}$ & 158 & $0.2245 \mathrm{c}$ & 6 & $0.0000 \mathrm{c}$ \\
\hline 113 & $1.3062 \mathrm{~b}$ & 105 & $0.5352 \mathrm{c}$ & 89 & $0.2175 \mathrm{c}$ & 21 & $0.0000 \mathrm{c}$ \\
\hline 124 & $1.2964 \mathrm{~b}$ & 15 & $0.5304 \mathrm{c}$ & 83 & $0.2149 \mathrm{c}$ & 23 & $0.0000 \mathrm{c}$ \\
\hline 57 & $1.2360 \mathrm{~b}$ & 119 & $0.5219 \mathrm{c}$ & 39 & $0.2131 \mathrm{c}$ & 27 & $0.0000 \mathrm{c}$ \\
\hline 68 & $1.2332 \mathrm{~b}$ & 163 & $0.5120 \mathrm{c}$ & 45 & $0.2127 \mathrm{c}$ & 28 & $0.0000 \mathrm{c}$ \\
\hline 167 & $1.2141 \mathrm{~b}$ & 107 & $0.5045 \mathrm{c}$ & 35 & $0.1951 \mathrm{c}$ & 30 & $0.0000 \mathrm{c}$ \\
\hline 162 & $1.1826 \mathrm{~b}$ & 26 & $0.5033 \mathrm{c}$ & 144 & $0.1949 \mathrm{c}$ & 42 & $0.0000 \mathrm{c}$ \\
\hline 127 & $1.1523 \mathrm{~b}$ & 108 & $0.5022 \mathrm{c}$ & 104 & $0.1855 \mathrm{c}$ & 62 & $0.0000 \mathrm{c}$ \\
\hline 147 & $1.1376 \mathrm{~b}$ & 17 & $0.4914 \mathrm{c}$ & 49 & $0.1710 \mathrm{c}$ & 65 & $0.0000 \mathrm{c}$ \\
\hline 10 & $1.1073 \mathrm{~b}$ & 66 & $0.4798 \mathrm{c}$ & 145 & $0.1605 \mathrm{c}$ & 70 & $0.0000 \mathrm{c}$ \\
\hline 148 & $1.0910 \mathrm{~b}$ & 24 & $0.4723 \mathrm{c}$ & 47 & $0.1572 \mathrm{c}$ & 77 & $0.0000 \mathrm{c}$ \\
\hline 76 & $1.0604 \mathrm{~b}$ & 73 & $0.4662 \mathrm{c}$ & 2 & $0.1565 \mathrm{c}$ & 78 & $0.0000 \mathrm{c}$ \\
\hline 12 & $1.0553 \mathrm{~b}$ & 44 & $0.4622 \mathrm{c}$ & 143 & $0.1463 \mathrm{c}$ & 82 & $0.0000 \mathrm{c}$ \\
\hline 116 & $1.0478 \mathrm{~b}$ & 8 & $0.4516 \mathrm{c}$ & 138 & $0.1457 \mathrm{c}$ & 84 & $0.0000 \mathrm{c}$ \\
\hline 164 & $1.0477 \mathrm{~b}$ & 19 & $0.4292 \mathrm{c}$ & 38 & $0.1404 \mathrm{c}$ & 86 & $0.0000 \mathrm{c}$ \\
\hline 121 & $1.0302 \mathrm{~b}$ & 1 & $0.4288 \mathrm{c}$ & 32 & $0.1372 \mathrm{c}$ & 87 & $0.0000 \mathrm{c}$ \\
\hline 126 & $1.0129 \mathrm{~b}$ & 31 & $0.4279 \mathrm{c}$ & 51 & $0.1350 \mathrm{c}$ & 88 & $0.0000 \mathrm{c}$ \\
\hline 169 & $0.9813 b$ & 3 & $0.4222 \mathrm{c}$ & 165 & $0.1322 \mathrm{c}$ & 91 & $0.0000 \mathrm{c}$ \\
\hline 136 & $0.9805 \mathrm{~b}$ & 14 & $0.4085 \mathrm{c}$ & 72 & $0.1230 \mathrm{c}$ & 93 & $0.0000 \mathrm{c}$ \\
\hline 146 & $0.9685 \mathrm{~b}$ & 109 & $0.3969 \mathrm{c}$ & 36 & $0.1220 \mathrm{c}$ & 94 & $0.0000 \mathrm{c}$ \\
\hline 130 & $0.9670 \mathrm{~b}$ & 157 & $0.3939 \mathrm{c}$ & 102 & $0.1190 \mathrm{c}$ & 95 & $0.0000 \mathrm{c}$ \\
\hline 120 & $0.9469 \mathrm{~b}$ & 101 & $0.3873 \mathrm{c}$ & 56 & $0.1180 \mathrm{c}$ & 96 & $0.0000 \mathrm{c}$ \\
\hline 117 & $0.9448 \mathrm{~b}$ & 53 & $0.3607 \mathrm{c}$ & 46 & $0.1153 \mathrm{c}$ & 97 & $0.0000 \mathrm{c}$ \\
\hline 135 & $0.9336 b$ & 20 & $0.3597 \mathrm{c}$ & 154 & $0.1120 \mathrm{c}$ & 98 & $0.0000 \mathrm{c}$ \\
\hline 128 & $0.9141 \mathrm{~b}$ & 140 & $0.3505 \mathrm{c}$ & 16 & $0.1056 \mathrm{c}$ & 99 & $0.0000 \mathrm{c}$ \\
\hline 159 & $0.9068 \mathrm{~b}$ & 79 & $0.3471 \mathrm{c}$ & 40 & $0.1015 \mathrm{c}$ & 100 & $0.0000 \mathrm{c}$ \\
\hline 25 & $0.9024 \mathrm{~b}$ & 11 & $0.3447 \mathrm{c}$ & 166 & $0.0982 \mathrm{c}$ & 137 & $0.0000 \mathrm{c}$ \\
\hline 142 & $0.9007 \mathrm{~b}$ & 22 & $0.3424 \mathrm{c}$ & 92 & $0.0934 \mathrm{c}$ & & \\
\hline 150 & $0.8992 \mathrm{~b}$ & 71 & $0.3363 \mathrm{c}$ & 103 & $0.0931 \mathrm{c}$ & & \\
\hline 129 & $0.8608 \mathrm{~b}$ & 160 & $0.3333 \mathrm{c}$ & 168 & $0.0911 \mathrm{c}$ & & \\
\hline
\end{tabular}

Means followed by the same letter in the column do not differ at 5\% significance by the Scott-Knott test. LIN: lineages; AFC: leaf area consumed $(\mathrm{cm})$

With regard to length of the caterpillars, a differentiated behavior of the lineages was observed in relation to the evaluation time (Table 6). The differences observed in relation to the average length in each evaluation period indicate that the caterpillars from the same posture were in different instars.

After five days feeding on lineages $21,86,87,100,161,94,88,62,78$ and 137 , there was a change in weight of larvae, besides a shorter length of the caterpillars. These were in the second instar with an average length ranging from 3 to $4 \mathrm{~mm}$, while the others were in development towards the third instar, with approximately $5.25 \mathrm{~mm}$. 
Table 5. Mean biomass of Spodoptera frugiperda fed during 5, 10 and 15 days with 28 corn genotypes.

\begin{tabular}{|c|c|c|c|c|}
\hline Lineages & Origin & $\begin{array}{l}\text { Mean biomass at } 5 \\
\text { days (mg) }\end{array}$ & $\begin{array}{l}\text { Mean biomass at } 10 \\
\text { days (mg) }\end{array}$ & $\begin{array}{l}\text { Mean biomass at } 15 \\
\text { days (mg) }\end{array}$ \\
\hline 4 & MPA & $0.00262 \mathrm{a} \mathrm{B}$ & 0.02865 a B & 0.19702 a A \\
\hline 6 & MPA & $0.00170 \mathrm{~b} B$ & 0.02745 a B & 0.20745 a A \\
\hline 21 & MPA & $0.00155 \mathrm{~b} \mathrm{~B}$ & 0.02907 a B & 0.19880 a A \\
\hline 23 & MPA & 0.00360 a C & $0.04550 \mathrm{a} \mathrm{B}$ & $0.20550 \mathrm{a} \mathrm{A}$ \\
\hline 27 & SIMPA & $0.00187 \mathrm{~b} \mathrm{~B}$ & 0.02177 a B & $0.16580 \mathrm{~b} \mathrm{~A}$ \\
\hline 28 & SIMPA & $0.00125 \mathrm{~b} \mathrm{~B}$ & 0.02990 a B & $0.13530 \mathrm{~b} \mathrm{~A}$ \\
\hline 30 & SIMPA & $0.00177 \mathrm{~b} B$ & 0.02650 a B & 0.17057 a A \\
\hline 62 & RVCERC & $0.00132 \mathrm{~b} \mathrm{~B}$ & 0.02142 a B & $0.15640 \mathrm{~b} \mathrm{~A}$ \\
\hline 65 & RVCERC & $0.00183 \mathrm{~b} \mathrm{~B}$ & 0.01966 a B & 0.17207 a A \\
\hline 70 & AG7088 & 0.00267 a C & $0.03732 \mathrm{a} \mathrm{B}$ & 0.25700 a A \\
\hline 74 & RVCERC & $0.00190 \mathrm{~b} \mathrm{~B}$ & 0.02215 a B & $0.14110 \mathrm{~b} \mathrm{~A}$ \\
\hline 77 & RVCERC & 0.00285 a C & 0.04030 a B & 0.24165 a A \\
\hline 78 & RVCERC & $0.00125 \mathrm{~b} \mathrm{~B}$ & 0.01223 a B & $0.10913 \mathrm{~b} \mathrm{~A}$ \\
\hline 86 & RVCERC & $0.00162 \mathrm{~b} \mathrm{~B}$ & 0.02267 a B & $0.10810 \mathrm{~b} \mathrm{~A}$ \\
\hline 87 & 2B587 & $0.00145 \mathrm{~b} \mathrm{~B}$ & 0.02317 a B & $0.14862 \mathrm{~b} \mathrm{~A}$ \\
\hline 88 & 2B587 & $0.00120 \mathrm{~b} B$ & 0.01980 a B & $0.16195 \mathrm{~b} A$ \\
\hline 91 & 2B587 & $0.00166 \mathrm{~b} \mathrm{~B}$ & 0.02203 a B & $0.13310 \mathrm{~b} \mathrm{~A}$ \\
\hline 94 & 2B587 & $0.00087 \mathrm{~b} \mathrm{~B}$ & 0.00576 a B & $0.05723 \mathrm{c} \mathrm{A}$ \\
\hline 96 & 2B587 & $0.00150 \mathrm{~b} \mathrm{~B}$ & 0.02636 a B & $0.16106 \mathrm{~b} \mathrm{~A}$ \\
\hline 97 & 2B587 & 0.00240 a B & $0.03112 \mathrm{a} \mathrm{B}$ & 0.20735 a A \\
\hline 98 & 2B587 & $0.00202 \mathrm{~b}$ B & 0.02845 a B & 0.17377 a A \\
\hline 99 & 2B587 & $0.00133 \mathrm{~b} \mathrm{~B}$ & 0.02596 a B & 0.18453 a A \\
\hline 100 & 2B587 & $0.00153 \mathrm{~b} \mathrm{~B}$ & $0.03030 \mathrm{a} \mathrm{B}$ & 0.19300 a A \\
\hline 110 & 2B587 & $0.00165 \mathrm{~b} \mathrm{~B}$ & 0.02292 a B & $0.14880 \mathrm{~b} \mathrm{~A}$ \\
\hline 123 & SUPERPRECOCE & 0.00215 a C & $0.03680 \mathrm{a} \mathrm{B}$ & 0.23400 a A \\
\hline 134 & 2B433 & $0.00230 \mathrm{a} \mathrm{B}$ & 0.01652 a B & $0.15442 \mathrm{~b} \mathrm{~A}$ \\
\hline 137 & RVCERC & $0.00000 \mathrm{~b} \mathrm{~B}$ & 0.00590 a B & 0.04920 c A \\
\hline 161 & AG7088 & $0.00142 \mathrm{~b} \mathrm{~B}$ & $0.02102 \mathrm{a} \mathrm{B}$ & $0.13370 \mathrm{~b} \mathrm{~A}$ \\
\hline
\end{tabular}

Scott-Knott test at $5 \%$ significance.

In studies carried out by Cruz (1995), in the second instar the length of the caterpillar body can reach up to $4.00 \mathrm{~mm}$ and in the third instar the length can vary from 6.35 to $6.50 \mathrm{~mm}$, which is in agreement with the present work. According to Lara (1991) and Davis et al. (1999), these changes observed in immature stages are characteristics observed in insects fed with genotypes that express antibiosis resistance, due to the presence of substances harmful to the biology of the pest, usually resulting in mortality. According to Vendramim and Guzzo (2009), in the first instars these effects have lethal consequences for the insect.

After 10 days of feeding, no significant differences were observed in larvae length confirming the same behavior between the treatments evaluated. This situation can be explained by two hypotheses, in which caterpillars that presented the best performance when fed for 5 days displayed a reduction in development when feeding continued, or caterpillars that were less developed were able to recover from a possible negative effect, provided by substances present in the resistant genotypes. According to Smith (2010) the longer duration of a developmental period of caterpillars can be observed due to the moderate expression of the antibiotic effects caused by resistant plants, resulting in a posterior reduction in weight and longevity of the insect. 
Table 6. Mean length of Spodoptera frugiperda larvae fed during 5, 10 and 15 days with 28 corn genotypes.

\begin{tabular}{|c|c|c|c|c|}
\hline Lineages & Origin & $\begin{array}{l}\text { Mean length at } 5 \\
\text { days }(\mathrm{mm})\end{array}$ & $\begin{array}{l}\text { Mean length at } 10 \\
\text { days }(\mathrm{mm})\end{array}$ & $\begin{array}{l}\text { Mean length at } 15 \\
\text { days }(\mathrm{mm})\end{array}$ \\
\hline 4 & MPA & 0.475 a C & $1.100 \mathrm{a} \mathrm{B}$ & $2.300 \mathrm{a} \mathrm{A}$ \\
\hline 6 & MPA & $0.425 \mathrm{a} \mathrm{C}$ & $1.000 \mathrm{a} \mathrm{B}$ & $2.275 \mathrm{a} \mathrm{A}$ \\
\hline 21 & HG49 & $0.400 \mathrm{~b} \mathrm{C}$ & $1.125 \mathrm{a} \mathrm{B}$ & $2.275 \mathrm{a} \mathrm{A}$ \\
\hline 23 & MPA & 0.525 a C & $1.225 \mathrm{a} \mathrm{B}$ & $2.375 \mathrm{a} \mathrm{A}$ \\
\hline 27 & SIMPA & 0.466 a C & $1.025 \mathrm{a} \mathrm{B}$ & $2.166 \mathrm{a} \mathrm{A}$ \\
\hline 28 & SIMPA & 0.450 a C & $1.300 \mathrm{a} \mathrm{B}$ & $2.000 \mathrm{~b} \mathrm{~A}$ \\
\hline 30 & SIMPA & 0.475 a C & $1.025 \mathrm{a} \mathrm{B}$ & $2.000 \mathrm{~b} \mathrm{~A}$ \\
\hline 62 & RVCERC & $0.350 \mathrm{~b} \mathrm{C}$ & $1.000 \mathrm{a} \mathrm{B}$ & $1.750 \mathrm{~b} \mathrm{~A}$ \\
\hline 65 & RVCERC & 0.433 a C & 0.925 a B & $2.175 \mathrm{a} \mathrm{A}$ \\
\hline 70 & AG7088 & 0.500 a C & 0.950 a B & $2.425 \mathrm{a} \mathrm{A}$ \\
\hline 74 & RVCERC & 0.450 a C & $1.000 \mathrm{a} \mathrm{B}$ & $2.050 \mathrm{~b} \mathrm{~A}$ \\
\hline 77 & RVCERC & 0.500 a C & $1.200 \mathrm{a} \mathrm{B}$ & $2.500 \mathrm{a} \mathrm{A}$ \\
\hline 78 & RVCERC & $0.350 \mathrm{~b} \mathrm{C}$ & 0.800 a B & $1.733 \mathrm{~b} \mathrm{~A}$ \\
\hline 86 & RVCERC & $0.400 \mathrm{~b} \mathrm{C}$ & 0.850 a B & $2.000 \mathrm{~b} \mathrm{~A}$ \\
\hline 87 & 2B587 & $0.400 \mathrm{~b} \mathrm{C}$ & 0.975 a B & $2.125 \mathrm{a} \mathrm{A}$ \\
\hline 88 & 2B587 & $0.350 \mathrm{~b} \mathrm{C}$ & 0.975 a B & $2.175 \mathrm{a} \mathrm{A}$ \\
\hline 91 & 2B587 & 0.433 a C & 0.925 a B & $1.833 \mathrm{~b} \mathrm{~A}$ \\
\hline 94 & 2B587 & $0.375 \mathrm{~b} \mathrm{~B}$ & 0.525 a B & $1.500 \mathrm{c} \mathrm{A}$ \\
\hline 96 & 2B587 & 0.433 a C & $1.125 \mathrm{a} \mathrm{B}$ & $1.900 \mathrm{~b} \mathrm{~A}$ \\
\hline 97 & 2B587 & 0.425 a C & $1.150 \mathrm{a} \mathrm{B}$ & $2.500 \mathrm{a} \mathrm{A}$ \\
\hline 98 & 2B587 & 0.475 a C & $1.150 \mathrm{a} \mathrm{B}$ & $2.125 \mathrm{a} \mathrm{A}$ \\
\hline 99 & 2B587 & 0.433 a C & $1.175 \mathrm{a} \mathrm{B}$ & $2.333 \mathrm{a} \mathrm{A}$ \\
\hline 100 & 2B587 & $0.400 \mathrm{~b} \mathrm{C}$ & $1.025 \mathrm{a} \mathrm{B}$ & $2.150 \mathrm{a} \mathrm{A}$ \\
\hline 110 & 2B587 & 0.425 a C & $1.100 \mathrm{a} \mathrm{B}$ & $1.850 \mathrm{~b} \mathrm{~A}$ \\
\hline 123 & SUPERPRECOCE & 0.450 a C & $1.100 \mathrm{a} \mathrm{B}$ & $2.433 \mathrm{a} \mathrm{C}$ \\
\hline 134 & $2 \mathrm{~B} 433$ & $0.450 \mathrm{a} \mathrm{c}$ & $1.000 \mathrm{a} \mathrm{B}$ & $2.000 \mathrm{~b} \mathrm{~A}$ \\
\hline 137 & RVCERC & $0.300 \mathrm{~b} \mathrm{C}$ & 0.800 a B & $1.450 \mathrm{c} \mathrm{A}$ \\
\hline 161 & AG7088 & $0.400 \mathrm{~b} \mathrm{C}$ & $1.125 \mathrm{a} \mathrm{B}$ & $2.150 \mathrm{~b} \mathrm{~A}$ \\
\hline
\end{tabular}

Besides leading to lower weights, the 94 lineages originated from the hybrid 2B587 and 137 from the RVCERC cultivar also influenced the lengths of caterpillars fed during 15 days. In this situation, it was observed that the caterpillars fed by these lineages were in fifth instar with approximately 1.5 centimeters in length, while the others were in sixth larval instar measuring up to 2.5 centimeters in length. According to Cruz (1995) the average length of the body of the armyworm in the fifth instar varies around 1.8 centimeters and in the sixth and last instar can reach up to 3.5 centimeters.

In research carried out by Paiva et al. (2016) and Williams et al. (1997), they proved that survival and larval growth are reduced with the feeding on resistant plants. Usually the larvae accumulate biomass until they reach the pre-pupal phase, when they consume biomass in order to harvest enough energy to transit to the pupal stage and stop feeding (Sá et al., 2009). Thus, the experiment was completed at the end of larval cycle of the caterpillar. The detection of these mechanisms of resistance involved in insect-host relationship contribute to the selection of plants and increase efficiency in breeding programs.

\section{CONCLUSIONS}

The no-choice non-feeding preference and antibiosis tests were efficient in the discrimination of promising lineages for maize breeding programs. Under laboratory 
conditions, the 94 2B587 hybrid originated lineages and 137 of RVCERC origin displayed a non-feeding preference and antibiosis mechanism of resistance.

\section{ACKNOWLEDGMENTS}

We thank the National Council for Scientific and Technological Development CNPQ for the Master's scholarship granted to the first author, the Goiás State Research Foundation - FAPEG and Federal University of Goiás - Jataí Regional for supporting the research performed.

\section{CONFLICTS OF INTEREST}

The authors declare no conflict of interest.

\section{REFERENCES}

Alvarez MP and Miranda Filho JB (2002). Diallel Crossing Among Maize Populations for resistance to fall armyworm. Sci. Agric. 59(4): 731-741. https://doi.org/10.1590/S0103-90162002000400017.

Barros EM, Torres JB and Bueno AF (2010). Oviposição, desenvolvimento e reprodução de Spodoptera frugiperda (J.E. Smith) (Lepidoptera: Noctuidae) em diferentes hospedeiros de importância econômica. Neotrop. Entomol. 39 (6): 996-1001. http://dx.doi.org/10.1590/S1519-566X2010000600023.

Boiça Júnior AL, Silva AG, Bottega DB, Rodrigues NEL, et al. (2011). Resistência de plantas e o uso de produtos naturais como táticas de controle no manejo integrado de pragas. In: Busoli AC, Fraga DF, Santos LC, Alencar JRCC, et al. (Ed) Tópicos em Entomologia Agrícola, Jaboticabal, São Paulo.

Cardoso WS, Pinheiro FA, Machado FP, Borges JTS, et al. (2011). Indústria do milho. In: Borém A, Rios SA. (Ed). Milho biofortificado, Visconde do Rio Branco, Minas Gerais.

Cruz I (1995). A lagarta-do-cartucho na cultura do milho. Embrapa Milho e Sorgo. Available in [http://www.infoteca.cnptia.embrapa.br/infoteca/handle/doc/475779]. Accessed May 21, 2020.

Cruz I (2008). Manejo de Pragas da Cultura do Milho. In: Cruz I. A cultura do milho. Embrapa Milho e Sorgo, Sete Lagoas, Minas Gerais.

Davis FM, Williams WP, Chang YM, Baker GT, et al. (1999). Differential growth of fall armyworm larvae (Lepidoptera: Noctuidae) reared on three phenotypic regions of whorl leaves from a resistant and a susceptible maize hybrid. Fla. Entomol. 82 (2): 248-254. DOI: 10.2307/3496576.

Falconer DS (1982). Introdução à genética quantitativa. $1^{\circ}$ edn. Viçosa, Minas Gerais.

Janini JC, Boica Junior AL, Jesus FG, Silva AG, et al. (2011). Effect of bean genotypes, insecticides, and natural products on the control of Bemisia tabaci (Gennadius) biotype B (Hemiptera: Aleyrodidae) and Caliothrips phaseoli (Hood) (Thysanoptera: Thripidae). Acta Sci. Agron. 33: 445-450. http://dx.doi.org/10.4025/actasciagron.v33i3.7577.

Jesus FG, Boica Junior AL, Alves GC, Busoli AC, et al. (2014). Resistance of cotton varieties to Spodoptera frugiperda. Rev. Colomb. Entomol. 40: 158-163. http://www.scielo.org.co/scielo.php?script=sci_arttext\&pid=S012004882014000200004.

Lara FM (1991). Princípios de resistência de plantas a insetos. $2^{\circ}$ edn. São Paulo.

Levin DA (1973). The role of trichomes in plant defence. Q. Rev. Biol. 48(1): 3-15.

Paiva la, Corrêa F, Silva CLT, Moura TL, Silva FC, Araújo MS and de Jesus FG. (2016). Resistance of corn genotypes to fall armyworm Spodoptera frugiperda (Lepidoptera: Noctuidae). Afr. J. Biotechnol. 15: 1877-1882. 10.5897/AJB2016.15399.

Ota EC, Lourenção AL, Duarte AP, Junior EUR, et al. (2011). Desempenho de cultivares de milho em relação à lagartado-cartucho. Bragantia. 70 (4): 850-859. http://dx.doi.org/10.1590/S0006-87052011000400018.

Pinto-Zevallos DM, Martins CBC and Pellegrino AC (2013). Compostos orgânicos voláteis na defesa induzida das plantas conta insetos herbivoros. Quim. Nova. 36 (9): 1395-1405. http://dx.doi.org/10.1590/S010040422013000900021.

Reis AJS and Miranda Filho JB (2003). Autocorrelação espacial na avaliação de compostos de milho para resistência à lagarta do cartucho (Spodoptera frugiperda). Pesq. Agropec. Trop. 33 (2): 65-72. DOI: 10.5216/pat.v33i2.2349.

R Core Team (2018). R: A language and environment for statistical computing. R Foundation for Statistical Computing, Vienna, Austria. Available in [http://www.R-project.org]. 
Rodrigues F, Pinto RGV, Albuquerque CJB, Filho EMF, et al. (2009). Capacidade de combinação entre linhagens de milho visando à produção de milho verde. Bragantia. 68 (1): 75-84. http://dx.doi.org/10.1590/S000687052009000100009.

Sá VGM, Fonseca BVC, Boregas KGB and Waquil JM (2009). Sobrevivência e desenvolvimento larval de Spodoptera frugiperda (J E Smith) (Lepidoptera: Noctuidae) em hospedeiros alternativos. Neotrop. Entomol. 38 (1): 108-115. http://dx.doi.org/10.1590/S1519-566X2009000100012.

Seifi A, Visser RGF and Bai Y (2013). How to effectively deploy plant resistances to pests and pathogens in crop breeding. Euphytica. 190 (3): 321-334. doi:10.1007/s10681-012-0823-9.

Shiferaw B, Boddupalli MP, Hellin J and Bänziger M (2011). Crops that feed the world 6. Past successes and future challenges to the role played by maize in global food security. Food Secur. 3 (3): 307-327. https://link.springer.com/article/10.1007/s12571-011-0140-5. Accessed May 25, 2017.

Silveira FT and Moro JR (2009). Utilização de linhagens parcialmente endogâmicas S3 para a obtenção de híbridos $\begin{array}{llllll}\text { simples de } & \text { milho. } & \text { Ver. } & \text { Biociências. } & 15 & \text { (2): }\end{array}$ http://periodicos.unitau.br/ojs/index.php/biociencias/article/view/1008/737.

Smith CM (2005). Plant resistance to arthropods: molecular and conventional approaches. pp 423.

USDA (United States Departament of Agriculture) (2018). World Agricultural Supply and Demand Estimates. IOP Publishing PhysicsWeb. Available in [https://www.usda.gov/oce/commodity/wasde/wasde0219.pdf]. Accessed May 01, 2020.

Vendramim JD and Guzzo EC (2009). Resistência de plantas e a bioecologia e nutrição dos insetos. In: Panizzi AR, Parra JRP (ed) Bioecologia e nutrição de insetos: base para o manejo integrado de pragas, Brasília, Distrito Federal.

Waquil MS, Pereira EJG, Carvalho SSS, Pitta RM, et al. (2016). Índice de adaptação e tempo letal da lagarta-do-cartucho em milho Bt. Pesq. Agropec. Bras. 51 (5): 563-570. http://dx.doi.org/10.1590/S0100204X2016000500017.

War AR, Paulraj MG, Ahmad T, Buhroo AA, et al. (2012). Mechanisms of plant defense against insect herbivores. Plant Signal Behav. 7 (10): 1306-1320. doi:10.4161/psb.2166.

Wiseman BR and Davis FM (1979) Plant resistance to the fall armyworm. Fla. Entomol. 62: 123-13o. DOI: $10.2307 / 3494088$.

Williams WP, Sagers JB, Hanten JA, Davis FM and Buckley PM (1997). Transgenic Corn Evaluated for Resistance to Fall Armyworm and Southwestern Corn Borer. Crop Sci. 37: 957-962 doi:10.2135/cropsci1997.0011183X003700030042x. 